\title{
Myocardial infarction after blunt chest trauma incurred during rugby football that later required cardiac transplantation
}

\author{
ANDREW H WATT, MICHAEL R STEPHENS \\ From the Department of Cardiology, University Hospital of Wales, Heath Park, Cardiff
}

SUMMARY A 28 year old man sustained an extensive anterolateral myocardial infarction five minutes after a blow on the chest while playing rugby football. The resulting persistent cardiac failure necessitated orthotopic cardiac transplantation.

Blunt trauma to the chest may result in various cardiac injuries, ${ }^{1}$ including myocardial infarction. Such cardiac injury is most commonly seen after road traffic accidents, due to impact of the chest on the steering wheel. Cardiac injury may also result from other types of blunt chest trauma. There are reports of such injuries occurring during sporting activities including cricket, ${ }^{2}$ association football, ${ }^{34}$ horse-riding, ${ }^{5}$ softball, ${ }^{6}$ boxing, ${ }^{7}$ and basketball. ${ }^{8}$ We report a case of myocardial infarction in a young man that occurred minutes after he received a blow on the chest while playing rugby. We have not found any other reports of a similar injury in which myocardial infarction occurred and was subsequently treated by cardiac transplantation.

\section{Case report}

A 28 year old man developed severe central chest pain associated with dyspnoea and sweating about five minutes after receiving a blow to the chest during a high tackle while playing rugby football. He was admitted to a local hospital where he was found to be hypotensive and to have pulmonary oedema. Myocardial infarction was diagnosed.

The next day he was transferred to this hospital because of evidence of persisting cardiogenic shock with left ventricular failure despite treatment with diuretics and intravenous inotropic support. On admission he was restless, sweating, had low volume pulses, and a blood pressure of $80 / 60 \mathrm{~mm} \mathrm{Hg}$. There

Requests for reprints to Dr A H Watt, Department of Pharmacology and Therapeutics, University of Wales College of Medicine, Heath Park, Cardiff CF4 4XN. was clinical and radiological evidence of severe pulmonary oedema. There was no evidence of pericardial fluid on echocardiography. Serial electrocardiograms showed the changes of an acute anterolateral myocardial infarction with reciprocal changes in the inferior limb leads (Fig. 1). Serial cardiac enzyme concentrations were considerably increased (peak creatine kinase being $8693 \mathrm{IU} / \mathrm{l}$ ). There was no sternal abnormality or sign of external injury.

Treatment with diuretics and intravenous and oral inotropes was continued and vasodilator treatment including angiotensin-converting enzyme inhibitors was added. Daily urine output was $650-1300 \mathrm{ml} / 24 \mathrm{~h}$ during the first week of admission. Mean pulmonary capillary wedge pressure during this period was in the range $14-20 \mathrm{~mm} \mathrm{Hg}$. Cardiac output was not measured. He slowly improved over about three weeks, although evidence of pulmonary oedema persisted. He had several episodes of pulmonary thromboembolism and was given anticoagulants. Recurrent episodes of ventricular tachycardia were successfully treated with amiodarone. He was discharged, at his own insistence, four weeks after admission. At that time the pulmonary component of the second heart sound was loud and he could walk less than 100 yards on the level.

Cardiac catheterisation was carried out eight weeks after the original admission; initially he had refused permission for invasive investigation. Despite intensive medical treatment his exercise tolerance remained very restricted. The loud pulmonary component of the second heart sound persisted and the pulmonary arterial pressure was found to be raised $(60 / 28 \mathrm{~mm} \mathrm{Hg})$, as was left atrial pressure (mean $28 \mathrm{~mm} \mathrm{Hg}$ ). Systemic arterial pres- 


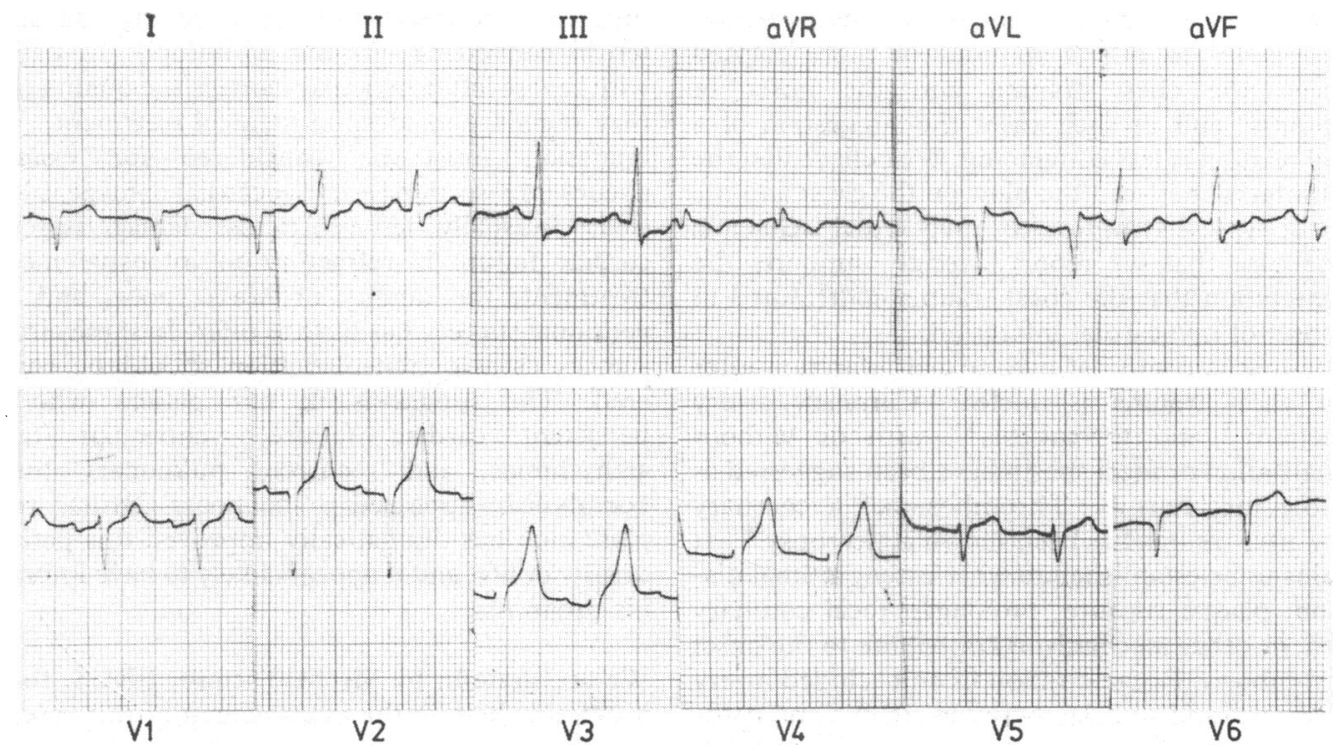

Fig. 1 Electrocardiogram showing evidence of an anterolateral myocardial infarction.

sure was $80 / 60 \mathrm{~mm} \mathrm{Hg}$. Left ventricular angiography demonstrated a considerably dilated ventricle, with widespread akinesis sparing only the septum and inferior wall. In the left coronary system there were several elongated stenoses, none of which

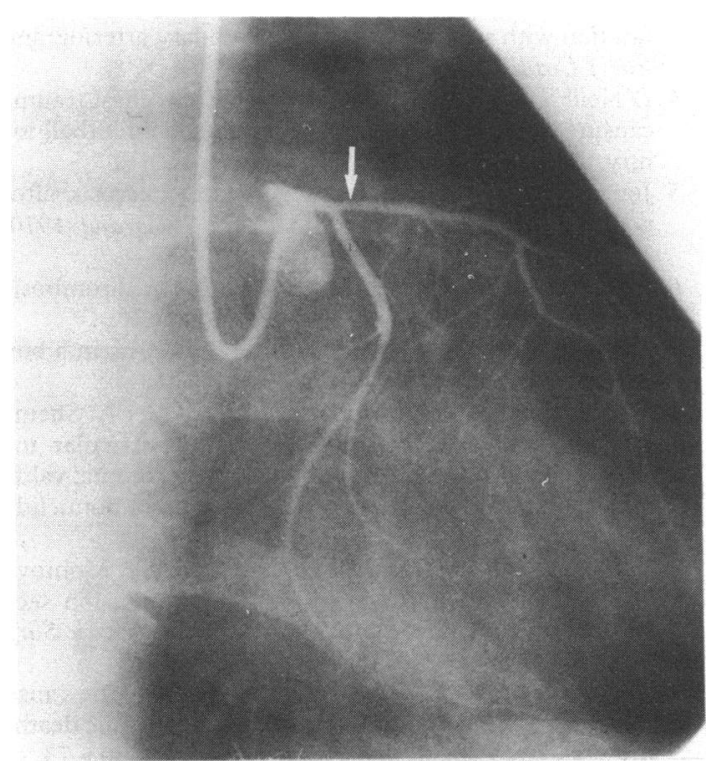

Fig. 2 Selective arteriography of the left coronary artery showing slight proximal stenosis (arrow) of the left anterior descending coronary artery and an elongated stenosis in the mid-portion of the circumflex coronary artery. appeared to be haemodynamically important. There was a stenosis of $<50 \%$ at the origin of the left anterior descending artery (Fig. 2). The dominant right coronary artery appeared to be normal.

He was referred to Harefield Hospital for consideration for cardiac transplantation in view of the continuing cardiac failure and symptomatic disability secondary to the extensive left ventricular damage. Orthotopic cardiac transplantation was carried out three months after the myocardial infarction.

Pathological examination of the excised heart showed extensive fibrosis of the left ventricle, sparing only the inferior wall and part of the septum. The left main coronary artery and proximal left anterior descending artery were atheromatous.

Twelve months after transplantation the patient has resumed full time employment and is progressing well.

\section{Discussion}

We are not aware of any other case report describing myocardial infarction after blunt chest trauma sustained while playing rugby football, nor do we know of any reported case in which myocardial infarction following blunt chest trauma of any cause has been treated by cardiac transplantation.

In cases of blunt chest trauma such as the one we describe it may be difficult to attribute with certainty the observed myocardial damage to the blow on the chest. In several cases only minutes passed between the time of the blow to the chest and the onset of 
cardiac ischaemic pain, ${ }^{34}$ and so a causal relation seems likely. In others an hour or more elapsed between the chest trauma and the onset of symptoms ${ }^{6}$ and in such cases the suggestion of a causal relation is less convincing. We believe that the short interval between the blow on the chest and the onset of typical symptoms of myocardial ischaemia in the case that we report strongly supports the interpretation that the chest trauma contributed to the onset of myocardial infarction.

The mechanism(s) of myocardial infarction after blunt chest trauma is unclear. Coronary artery dissection $^{27}$ and thrombosis ${ }^{36}$ with or without recanalisation or focal spasm have been suggested as possible mechanisms. Thrombosis of a coronary artery after trauma has been shown at necropsy. ${ }^{6}{ }^{7}$ It remains to be established to what extent intimal dissection, plaque rupture, prolonged focal coronary spasm, or other mechanisms contribute to the onset of thrombosis. Recanalisation of an occluded artery may occur, ${ }^{9}$ and so delay between trauma and coronary arteriography may falsely increase the number of cases with "normal" coronary arteries at angiography. In some cases myocardial contusion rather than coronary artery injury may have occurred. It appears likely that different mechanisms may operate in individual patients.

In our case we believe the most likely sequence of events was that thrombotic occlusion occurred at the origin of the left anterior descending artery after the blow on the chest and that recanalisation occurred before coronary arteriography was carried out. It may be that the blow on the chest caused fissuring of an atherosclerotic plaque and secondary thrombosis, similar to the "spontaneous" fissuring of plaques believed to occur frequently in ischaemic heart disease. ${ }^{10}$

Management of patients sustaining a myocardial infarction after blunt chest trauma must be empirical in view of the small number of reported cases. Initially it seems reasonable to manage the situation much as one would when myocardial infarction is secondary to coronary atheroma. Echocardiographic examination of the heart for other abnormalities secondary to trauma-for example haemopericardium-appears to be appropriate.

Many patients will make an uneventful recovery after their myocardial infarct but some may have residual angina pectoris. ${ }^{4}$ This may be due to incorporation of mural thrombus into the coronary arterial wall which results in a haemodynamically important stenosis. It seems reasonable to treat such angina pectoris in the conventional manner.

Other patients may have cardiac failure which responds to medical treatment with diuretics, digoxin, and vasodilators (particularly the angiotensin converting enzyme inhibitors). In patients with severe left ventricular damage the quality of life may remain poor despite medical treatment, ${ }^{11}$ and operation must be considered-for example, aneurysm resection ${ }^{9}$-if myocardial damage is localised. Unusually, left ventricular damage is severe, cardiac failure is refractory to intensive medical treatment, the quality of life is poor, and conventional surgery has little to offer. In these circumstances cardiac transplantation should be considered. The prognosis in our patient who had persistent cardiac failure, ventricular tachyarrhythmias, and recurrent pulmonary thromboembolism appeared to be poor. Cardiac transplantation has dramatically improved this patient's quality of life, and it appears likely to have improved his prognosis.

We are grateful to Mr M Yacoub, FRCS, for his invaluable help in the management of this case.

\section{References}

1 Parmley LF, Manion WC, Mattingly TW. Nonpenetrating traumatic injury of the heart. Circulation 1958; 18: 371-96.

2 Rab SM. Traumatic myocardial infarction. $\mathrm{Br} f \mathrm{Clin}$ Pract 1969; 23: 172-3.

3 de Feyter PJ, Roos JP. Traumatic myocardial infarction with subsequent normal coronary arteriogram. Eur $\mathcal{f}$ Cardiol 1977; 6: 25-31.

4 O'Neill S, Walker F, O'Dwyer WF. Blunt chest trauma causing myocardial infarction - an unusual football injury. Ir Med f 1981; 74: 138.

5 Jones FL Jr. Transmural myocardial necrosis after nonpenetrating cardiac trauma. Am $\mathcal{F}$ Cardiol 1970; 26: 419-22.

6 MacDonald D. Repeated and fatal coronary thrombosis in a young man. $\mathscr{f} A M A 1941$; 116: 2846-8.

7 Jokl E, Greenstein J. Fatal coronary sclerosis in a boy of ten years. Lancet 1944; ii: 659.

8 Motro M, Barzilay Z, Schneeweiss A, Battler A, ShemTov A, Neufeld HN. Isolated right ventricular infarction and aneurysm due to blunt chest trauma: value of two-dimensional echocardiography and radionuclide angiography. Am Heart $\mathcal{F}$ 1981; 101: 679-80.

9 Pifarre R, Grieco J, Garibaldi A, Sullivan HJ, Montoya A, Bakhous M. Acute coronary artery occlusion secondary to blunt chest trauma. $\mathcal{F}$ Thorac Cardiovasc Surg 1982; 83: 122-5.

10 Davies MJ, Thomas AC. Plaque fissuring - the cause of acute myocardial infarction, sudden ischaemic death, and crescendo angina. Br Heart $\mathcal{f}$ 1985; 53: 363-73.

11 Candell J, Valle V, Paya J, Cortadellas J, Esptugas E, Rius J. Post-traumatic coronary occlusion and early left ventricular aneurysm. Am Heart $\mathcal{f}$ 1979; 97: 509-12. 